Full Length Article

\title{
Isometric pre-conditioning blunts exercise-induced muscle damage but does not attenuate changes in running economy following downhill running
}

\author{
Leonardo C.R. Lima ${ }^{\text {a,b,c,*, Natália M. Bassan }}{ }^{\text {, }}$ Adalgiso C. Cardozo ${ }^{\text {, }}$, \\ Mauro Gonçalves $^{\mathrm{d}}$, Camila C. Greco ${ }^{\mathrm{a}}$, Benedito S. Denadai ${ }^{\mathrm{a}}$ \\ ${ }^{a}$ Human Performance Laboratory, São Paulo State University, Av 24-A, 1515, Rio Claro, SP 13506-900, Brazil \\ ${ }^{\mathrm{b}}$ Faculty of Biological and Health Sciences, Centro Universitário Hermínio Ometto, Av. Maximiliano Baruto, 500, Araras, SP 13607-339, Brazil \\ ${ }^{\mathrm{c}}$ Faculty of Physical Education, Centro Universitário Salesiano de São Paulo, Rua Baronesa Geraldo Resende, 330, Campinas, SP 13075-270, Brazil \\ d Biomechanics Laboratory, São Paulo State University, Av 24-A, 1515, Rio Claro, SP 13506-900, Brazil
}

\section{A R T I C L E I N F O}

\section{Keywords:}

Exercise prophylaxis

Maximal voluntary contractions

Myofibrilar disruption

Running efficiency

Decline running

Eccentric exercise

\begin{abstract}
A B S T R A C T
Running economy (RE) is impaired following unaccustomed eccentric-biased exercises that induce muscle damage. It is also known that muscle damage is reduced when maximal voluntary isometric contractions (MVIC) are performed at a long muscle length 2-4 days prior to maximal eccentric exercise with the same muscle, a phenomenon that can be described as isometric preconditioning (IPC). We tested the hypothesis that IPC could attenuate muscle damage and changes in RE following downhill running. Thirty untrained men were randomly assigned into experimental or control groups and ran downhill on a treadmill $(-15 \%)$ for 30 min. Participants in the experimental group completed 10 MVIC in a leg press machine two days prior to downhill running, while participants in the control group did not perform IPC. The magnitude of changes in muscle soreness determined $48 \mathrm{~h}$ after downhill running was greater for the control group $(122 \pm 28 \mathrm{~mm})$ than for the experimental group $(92 \pm 38 \mathrm{~mm})$. Isometric peak torque recovered faster in the experimental group compared with the control group (3 days vs. no full recovery, respectively). No significant effect of IPC was found for countermovement jump height, serum creatine kinase activity or any parameters associated with RE. These results supported the hypothesis that IPC attenuates changes in markers of muscle damage. The hypothesis that IPC attenuates changes in RE was not supported by our data. It appears that the mechanisms involved in changes in markers of muscle damage and parameters associated with RE following downhill running are not completely shared.
\end{abstract}

\section{Introduction}

Running economy (RE) is defined as the energy demand to sustain running at a given submaximal speed (Assumpção, Lima, Oliveira, Greco, \& Denadai, 2013). As an important predictor of long-distance running performance, RE can determine which of two athletes with similar maximal oxygen uptake $\left(\mathrm{VO}_{2} \mathrm{max}\right)$ could win a long-distance race (Hoogkamer, Kipp, Spiering, \& Kram, 2016). The association between neuromuscular/biomechanical variables and RE is not yet fully understood and has been the object of many

\footnotetext{
* Corresponding author at: Human Performance Laboratory, São Paulo State University, Avenida 24-A, 1515, 13506-900 Rio Claro, SP, Brazil.

E-mail addresses: leonardocrlima@gmail.com (L.C.R. Lima), adalgiso@rc.unesp.br (A.C. Cardozo), grecocc@rc.unesp.br (C.C. Greco), bdenadai@rc.unesp.br (B.S. Denadai).
} 
studies (Lacour \& Bourdin, 2015; Saunders, Pyne, Telford, \& Hawley, 2004). For instance, it is well established that maximal strength and power training regimens improve RE in experienced runners (Saunders et al., 2006; Turner, Owings, \& Schwane, 2003; Støren, Helgerud, Støa, \& Hoff, 2008; Denadai, de Aguiar, Lima, Greco, \& Caputo, 2017).

The relationship between RE and muscle function has been further investigated in studies using eccentric exercise-induced muscle damage (EIMD) as a research model (Assumpção et al., 2013). EIMD is a multifactorial phenomenon that occurs when skeletal muscle is exposed to mechanical stress imposed by unaccustomed eccentric contractions (Clarkson \& Hubal, 2002). This exposure to muscle strain leads to transient decreases in muscle function, delayed onset muscle soreness (DOMS), and leakage of intracellular proteins such as creatine kinase (CK) to the blood stream (Brentano \& Martins Kruel, 2011). It has also been shown that EIMD reduces explosive force production (Peñailillo, Blazevich, Numazawa, \& Nosaka, 2015), and range of motion (Chen, Lin, Chen, Lin, \& Nosaka, 2011), and impairs RE (Braun \& Paulson, 2012; Chen, Nosaka, \& Tu, 2007). Indeed, Chen, Nosaka, et al. (2007) found that markers of EIMD and RE were affected following a 30-min downhill run in college-level soccer players, but the magnitude of impairment in RE (6.9\% increase in oxygen uptake) was lesser than the decrease in maximal voluntary torque production $(-21.3 \%)$. Additionally, RE recovered faster than muscle strength ( 3 vs 4 days, respectively).

When a damaging bout is performed for a second time, changes in markers of EIMD are attenuated. This partial protection has been referred to as the repeated bout effect (Hyldahl, Chen, \& Nosaka, 2017). Apparently, the repeated bout effect also occurs for RE parameters. Chen, Chen, et al. (2007) showed that changes in EIMD, RE, and running kinematic parameters were considerably smaller following a second bout of downhill running when compared to changes induced by an initial downhill run performed 5 days earlier. Furthermore, Doma et al. (2017) showed that changes in indirect markers of EIMD and submaximal running performance are further blunted following a third bout of damaging exercise (resistance training at 6 repetition maximum). Even though the repeated bout effect might be considered an efficient protective strategy, the damage induced by the first bout might be considered detrimental in many contexts (Lima \& Denadai, 2015). Thus, an alternative strategy that does not require initial damage is of great relevance.

There is evidence that performing low-volumes (2-10) of maximal voluntary isometric contractions (MVIC) 2-4 days prior to damaging bouts promotes significant attenuation of markers of EIMD in the ipsilateral (Chen, Nosaka, Peake, \& Chen, 2012; Chen et al., 2013) and contralateral arm (Chen et al., 2018). Thus, isometric pre-conditioning (IPC) could be an efficient non-damaging alternative to promote acute protection against EIMD. However, it is important to note that the prophylactic effect of IPC was investigated mostly in upper limb muscles, which are not frequently exposed to EIMD. In this context, Lima and Denadai (2015) proposed that lower limb muscles, which are highly exposed to EIMD, would present a reduced protective effect during eccentric exercise. To date, the only study that has investigated the effects of IPC on signs and symptoms of EIMD in lower-limb muscles was performed by Tseng et al. (2016). A potent protective effect was identified when subjects performed 60 MVIC two weeks prior to maximal eccentric contractions in an isokinetic dynamometer. However, the authors found small, yet significant, changes in markers of EIMD following the $60 \mathrm{MVIC}$, which seems to be contradictory to the principle of IPC as a non-damaging alternative.

To the best of our knowledge, no previous studies have investigated the effects of low-volume IPC on changes in markers of EIMD following downhill running. Nor have we found evidence showing that this type of pre-conditioning exercise attenuates the magnitude of changes in RE following damaging bouts of any sort. Thus, the aim of the present study was to investigate the effects of performing an IPC bout consisting of 10 closed kinetic chain MVIC two days prior to a downhill running bout on: 1) muscle function; 2) indirect markers of EIMD; 3) metabolic and kinematic parameters of RE. It was hypothesized that the IPC protocol would attenuate changes in markers of EIMD and parameters of RE in the following days.

\section{Methods}

\subsection{Participants}

Thirty young men $(22.8 \pm 2.3$ years, $1.77 \pm 0.04 \mathrm{~m}, 78.6 \pm 8.9 \mathrm{~kg})$ with no recent $(6 \mathrm{months})$ experience with strength and/or endurance training volunteered to participate in the present study. They had no history of muscular, joint or bone injuries in the lower limbs. Sample size was calculated based on an effect size of 0.5 , and $\alpha$ level of 0.05 , and $\beta$ level of 0.8 . Inclusion criteria for this study were healthy men aged 18-30 years old. None of the participants reported regular use of any type of medicine or nutritional supplement before and during the study upon questioning from the examiners. Participants provided written consent for their participation on the study and were instructed to maintain their daily routines, not engaging in novel exercise programs and maintaining their regular eating habits. All procedures were conducted in accordance with the Helsinki Declaration on the use of humans as research subjects, and the protocol was approved by the University's Ethics Committee.

\subsection{Experimental design}

Incremental tests were performed on a treadmill (Pulsar, $\mathrm{h} / \mathrm{p} / \operatorname{cosmos}$, Germany) prior to any experimental procedure to determine the maximal oxygen uptake $\left(\mathrm{VO}_{2} \mathrm{max}\right)$ and velocity associated with $\mathrm{VO}_{2} \mathrm{max}\left(\mathrm{vVO}_{2} \mathrm{max}\right)$ using a gas analyzer (Quark, PFT Ergo, Cosmed, Italy). The incremental test protocol has been described elsewhere (Lima, Assumpção, Prestes, \& Denadai, 2015). Following the determination of $\mathrm{VO}_{2}$ max, participants were randomly assigned to either a control $(\mathrm{n}=15)$ or experimental $(\mathrm{n}=15)$ group. Participants in both groups performed a 30-min downhill $(-15 \%)$ running bout at a speed of $70 \%$ of their $\mathrm{VO}_{2} \mathrm{max}$. $\mathrm{Muscle}$ function (isometric peak torque and countermovement jump height) was assessed before, immediately after, and 1-4 days following downhill running. Muscle soreness was assessed immediately before and 1-4 days following downhill running. Serum CK activity was assessed only before, and 2 and 4 days after the run due to the invasiveness of the procedures adopted for blood extraction (i.e., from 
the participant's earlobes). RE parameters (oxygen uptake, minute ventilation, blood lactate concentration, rate of perceived effort and stride frequency) were assessed before, immediately after, and 1-4 days following downhill running.

\subsection{Isometric pre-conditioning}

Participants in the experimental group performed 10 MVIC in a leg press machine two days prior to the downhill run as an IPC protocol. Participants in the control group remained inactive during the pre-conditioning period of the experiment. A two-day interval between the IPC bout and the downhill running bout was chosen based on data from Chen et al. (2013), which reported that the magnitude of the protective effect induced by IPC is optimal at this interval. A leg press machine was adopted so that participants performed closed kinetic chain MVIC during the IPC protocol, which are similar to the closed kinetic chain eccentric contractions performed while running downhill (Assumpção et al., 2013). Each pre-conditioning MVIC lasted 3 s, with 45-s intervals between them, as proposed by Chen, Nosaka, et al. (2012). To ensure that the quadriceps muscle group was in a stretched state during the MVIC, the machine seat was adjusted so that the participants' knee joints were positioned at a $100^{\circ}$ angle (considering full extension as $0^{\circ}$ ).

\subsection{Criterion measures}

\subsubsection{Running economy}

$\mathrm{RE}$ tests consisted of 5-min runs at $\mathbf{8 0} \% \mathrm{VO}_{2} \mathrm{max}$, since Chen, Nosaka, Lin, Chen, and Wu (2009) showed that RE is predominantly affected by downhill running-induced muscle damage when assessed at relatively high intensities. Breath-by-breath gas exchanges were recorded during RE tests. Mean oxygen uptake and minute ventilation values recorded at the last minute of each test were used for analyses (Chen, Chen, et al., 2007). The last minute of each RE test was filmed (Compact Full HD Memory Camcorder, Samsung, South Korea) to determine stride length (SL) and step frequency (SF) (Chen, Nosaka, et al., 2007). A sample of blood (25 $\mu$ ) was collected from participant's earlobes immediately after RE tests to determine blood lactate concentration (YSL 2300 STAT, Yellow Springs, USA). Rating of perceived exertion was also assessed immediately after the test using the Borg scale (Borg, 1970).

\subsubsection{Isometric peak torque}

Isometric peak torque was determined in an isokinetic dynamometer (System 3, Biodex Systems, USA). Participants were seated on a chair with their hips flexed $\left(85^{\circ}\right)$ and had their trunks, waist and thighs firmly secured by belts. Dynamometer settings were recorded for each participant and reproduced during all experimental sessions. After a warm-up consisting of three single-legged submaximal isometric contractions of the knee extensors, participants were instructed to perform two 3-s MVIC as forcefully as they could with their knee joint at $75^{\circ}$ flexion (full knee extension $=0^{\circ}$ ). A 180-s rest interval was given between contractions. Isometric peak torque was considered as the greatest torque value obtained from both contractions. Participants received strong verbal encouragement to perform the MVIC.

\subsubsection{Countermovement jump height}

Countermovement jump height was assessed using a force plate with a sample frequency of 2000 Hz (OR6-6, AMTI, USA). Participants stood on the force plate and were informed to jump as high as they could after performing a rapid squat and to land on the force plate. The depth of the squat was self-selected. Participants performed two familiarization sessions on separate days before the assessment of pre-downhill running countermovement jump height. Two countermovement jumps were performed by each participant with a rest interval of $180 \mathrm{~s}$ in between. Jump height was calculated based on the recorded flight time using the formula: $\mathbf{J H}=1 / 2(\mathbf{f t} / \mathbf{2})^{\mathbf{2}} \cdot \mathbf{g}$, where $\mathbf{J H}$ is jump height, $\mathbf{f t}$ is flight time, and $\mathbf{g}$ is the acceleration of gravity (9.81 $\mathrm{m} \cdot \mathrm{s}^{2}$ ) (Dias et al., 2011). The highest jump was used for analyses.

\subsubsection{Muscle soreness}

Muscle soreness was assessed while performing a stepping test. Participants slowly stepped up and down from a $45 \mathrm{~cm}$ chair and filled a $100 \mathrm{~mm}$ visual analog scale with the sayings "not sore" and "very, very sore" at the extremities. They were instructed to report the soreness they felt in their plantar flexors, knee extensors, and hip extensors. The sum of the reported values was considered for analyses.

\subsubsection{Serum CK activity}

Approximately $500 \mu \mathrm{l}$ of blood were collected from participants' earlobes and centrifuged (Microhemato Modelo 2410, Fanem, Brazil). Following centrifugation, serum was separated from plasma and stored at $-70^{\circ} \mathrm{C}$ for analyses of serum CK activity in a spectrophotometer (Bio-2000, Bioplus, Brazil) using a commercial kit (CK-NAC UV, Wiener Lab, Argentina). Reference values for this kit ranges from 24 to $195 \mathrm{U} / \mathrm{l}$.

\subsection{Statistical analyses}

Data normality was assessed by a Shapiro-Wilk tests. The homogeneity and sphericity assumptions were assessed by the Levene and Mauchly tests, respectively. Changes over time and between groups were tested using mixed-design two-way ANOVA followed by Bonferroni's post hoc tests. Significance level was set as $\mathrm{p}<0.05$. Data is expressed as mean \pm standard deviation, unless otherwise 
Table 1

Anthropometric data and performance in incremental tests.

\begin{tabular}{lll}
\hline & CON $(\mathrm{n}=15)$ & IPC $(\mathrm{n}=15)$ \\
\hline Age (years) & $23.4(2.4)$ & $22.3(2.1)$ \\
Body mass $(\mathrm{kg})$ & $79.2(7.1)$ & $77.9(10.6)$ \\
Stature $(\mathrm{m})$ & $1.77(0.05)$ & $1.78(0.05)$ \\
Body mass index $\left(\mathrm{kg} / \mathrm{m}^{2}\right)$ & $25.3(2)$ & $24.6(2.3)$ \\
$\mathrm{VO}_{2} \max (\mathrm{ml} / \mathrm{kg} / \mathrm{min})$ & $42.5(4.1)$ & $43.7(3.1)$ \\
$\mathrm{vVO}_{2} \max (\mathrm{km} / \mathrm{h})$ & $14(0.8)$ & $14.6(1.0)$ \\
$\mathrm{V}_{\text {Downhill }}(\mathrm{km} / \mathrm{h})$ & $9.8(0.6)$ & $10.2(0.7)$ \\
$\mathrm{V}_{\mathrm{RE}}(\mathrm{km} / \mathrm{h})$ & $10.2(0.8)$ & $10.3(1.0)$ \\
\hline
\end{tabular}

Values are means ( $\pm \mathrm{SD}$ ). CON: control group; IPC: isometric pre-conditioning group; $\mathrm{VO}_{2} \max$ : $\max -$ imal oxygen uptake; $\mathrm{v} V O 2 m a x$ : speed at maximal oxygen uptake; $\mathrm{V}_{\text {Downhill: }}$ speed used for downhill running; $\mathrm{V}_{\mathrm{RE}}$ : speed used during running economy tests.

stated.

\section{Results}

Participants' mean age, stature, body mass, body mass index, $\mathrm{VO}_{2} \mathrm{max}, \mathrm{vVO}_{2} \mathrm{max}$, downhill running velocity and $\mathrm{RE}$ velocity were not different between groups and are represented as means \pm SD in Table 1.

Significant effects of time $(\mathrm{p}<0.01 ; \mathrm{F}=136)$ and group $v s$. time interaction $(\mathrm{p}=0.05 ; \mathrm{F}=2.28)$ were found for isometric peak torque (Fig. 1A). No significant effect of group was found for this criterion measure. Isometric peak torque significantly decreased immediately following downhill running and remained reduced 1-4 days after it for the control group. Isometric peak torque decreased immediately after the downhill run for the experimental group, but returned to baseline values three days after. A significant effect of time ( $\mathrm{p}<0.01 ; \mathrm{F}=39.45)$ was identified for countermovement jump height (CMJ) (Fig. 1B), which decreased ( $\mathrm{p}<0.05)$
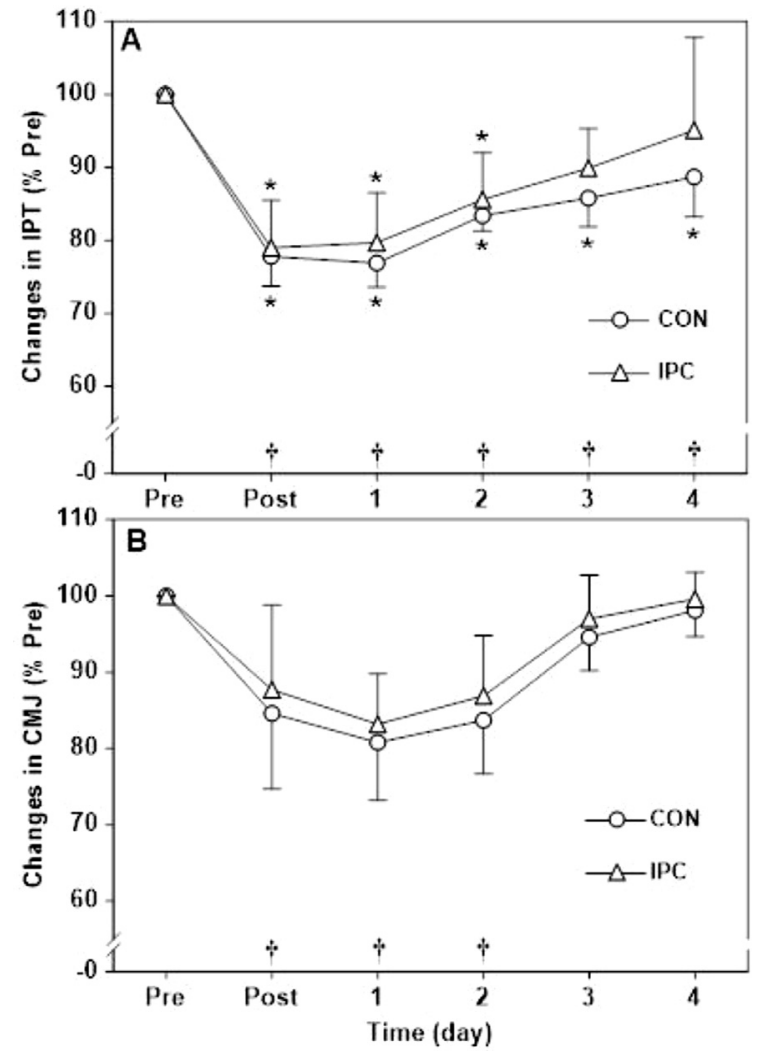

Fig. 1. Changes over time in isometric peak torque (IPT) (A) and countermovement jump height (CMJ) (B) for both groups. The isometric preconditioning group (IPC) performed maximal isometric contractions two days prior to downhill running while the control group (CON) did not perform any pre-conditioning. *Values are significantly different $(\mathrm{p}<0.01)$ as compared to pre downhill running values for the same group. ${ }^{\dagger}$ $\mathrm{p}<0.05$ compared to baseline values for both groups together. 


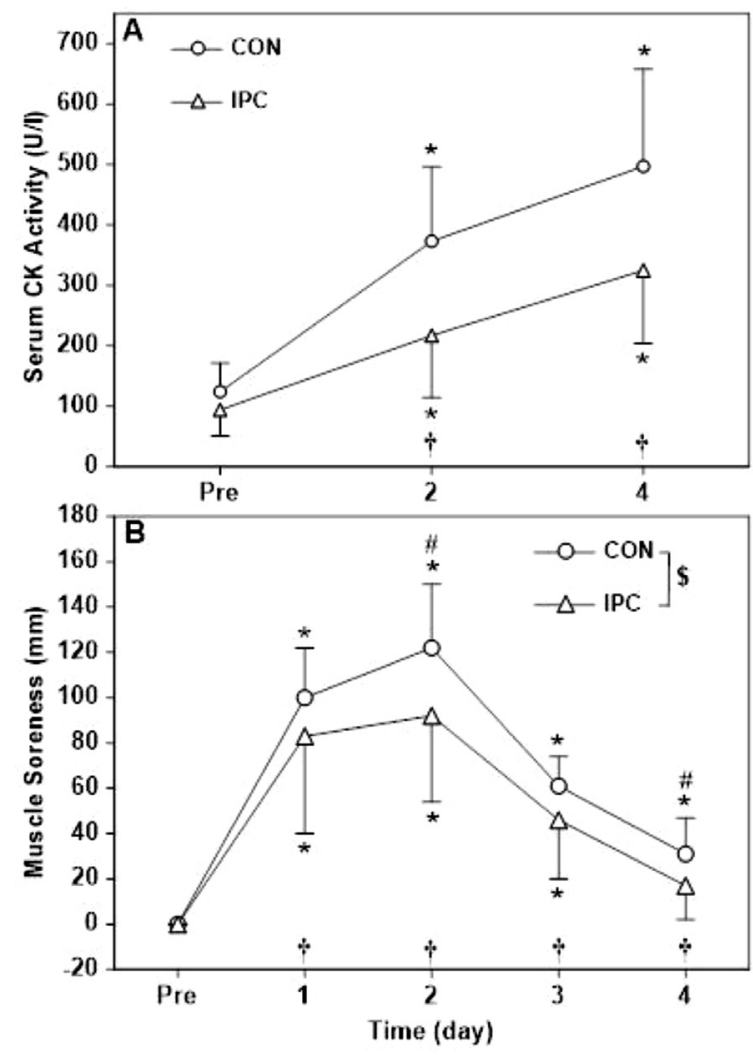

Fig. 2. Changes over time in serum creatine kinase (CK) activity (A) and muscle soreness (B). The isometric pre-conditioning group (ISO) performed maximal isometric contractions two days prior to downhill running while the control group (CON) did not perform any pre-conditioning. "Values are significantly different $\left(\mathrm{p}<0.01\right.$ ) as compared to pre downhill running values for the same group; ${ }^{*} \mathrm{p}<0.05$ compared to the experimental group at the same point in time; ${ }^{\dagger} \mathrm{p}<0.05$ compared to baseline values for both groups together; $\$$ significant effect of groups.

immediately after downhill running and fully recovered three days after. No significant effects of group or group vs. time interaction were found for CMJ. IPT and CMJ are represented in Fig. 1.

Significant effects of time ( $<<0.01 ; \mathrm{F}=187.6)$, group $(\mathrm{p}=0.029 ; \mathrm{F}=5.28)$, and groups $v s$. time interaction ( $\mathrm{p}=0.036$; $\mathrm{F}=2.66$ ) were found for muscle soreness following downhill running (Fig. 2B). Perceived soreness increased ( $\mathrm{p}<0.01$ ) one day after downhill and remained altered throughout the entire study period for the control group, while full recovery was reached four days after downhill running for the experimental group. Soreness was greater $(p<0.01)$ for the control group two and four days after downhill running.

Serum creatine kinase (CK) activity presented a significant effect of time ( $\mathrm{p}<0.01 ; \mathrm{F}=95.83$ ) but no effects of group or group vs. time interaction were flagged (Fig. 2A). Serum CK activity increased ( $<<0.01$ ) two days after downhill running and peaked 4 days after and was significantly greater 4 days after downhill running when compared to 2 days for both groups (Fig. 2).

Significant effects of time were identified for oxygen uptake $\left(\mathrm{VO}_{2}: \mathrm{p}<0.01 ; \mathrm{F}=301.3-\right.$ Fig. 3A), ventilation (VE: $\mathrm{p}<0.01$; $\mathrm{F}=118.1$ - Fig. 3B), blood lactate concentration (Lac: $\mathrm{p}<0.01 ; \mathrm{F}=57.9-$ Fig. 3C), rate of perceived exertion (RPE: $\mathrm{p}<0.05$; $\mathrm{F}=154.8-$ Fig. 3D), stride length (SL: $\mathrm{p}<0.01 ; \mathrm{F}=32.5-$ Fig. 3E), and stride frequency (SF: $\mathrm{p}<0.01 ; \mathrm{F}=31.5-\mathrm{Fig} .3 \mathrm{~F}$ ) during level running after the downhill running bout. $\mathrm{VO}_{2}, \mathrm{VE}$ and $\mathrm{Lac}$ increased $(\mathrm{p}<0.01$ ) immediately after downhill running and remained altered on the following two days. RPE increased $(\mathrm{p}<0.01)$ after downhill running and remained elevated on the following three days. Running kinematic parameters (SL and SF) presented a significant effects of time (p $<0.05)$, showing significant changes immediately after the downhill running and fully recovered three days after. No significant effects of group or group $v s$. time interaction were identified for any RE marker in the present study (Fig. 3).

\section{Discussion}

The purpose of this study was to test the hypotheses that performing a low-volume pre-conditioning bout consisting of 10 MVIC two days prior to a downhill running bout would attenuate the magnitude of changes in muscle function, markers of EIMD and parameters associated with RE. Consistent with previous research (Chen, Nosaka, et al., 2007; Chen et al., 2009), it was verified that downhill running elicited significant alterations in both EIMD and RE parameters. However, the main and original findings were as follows: (1) IPC leads to partial protection against EIMD, blunting changes in muscle soreness and promoting faster recovery from 

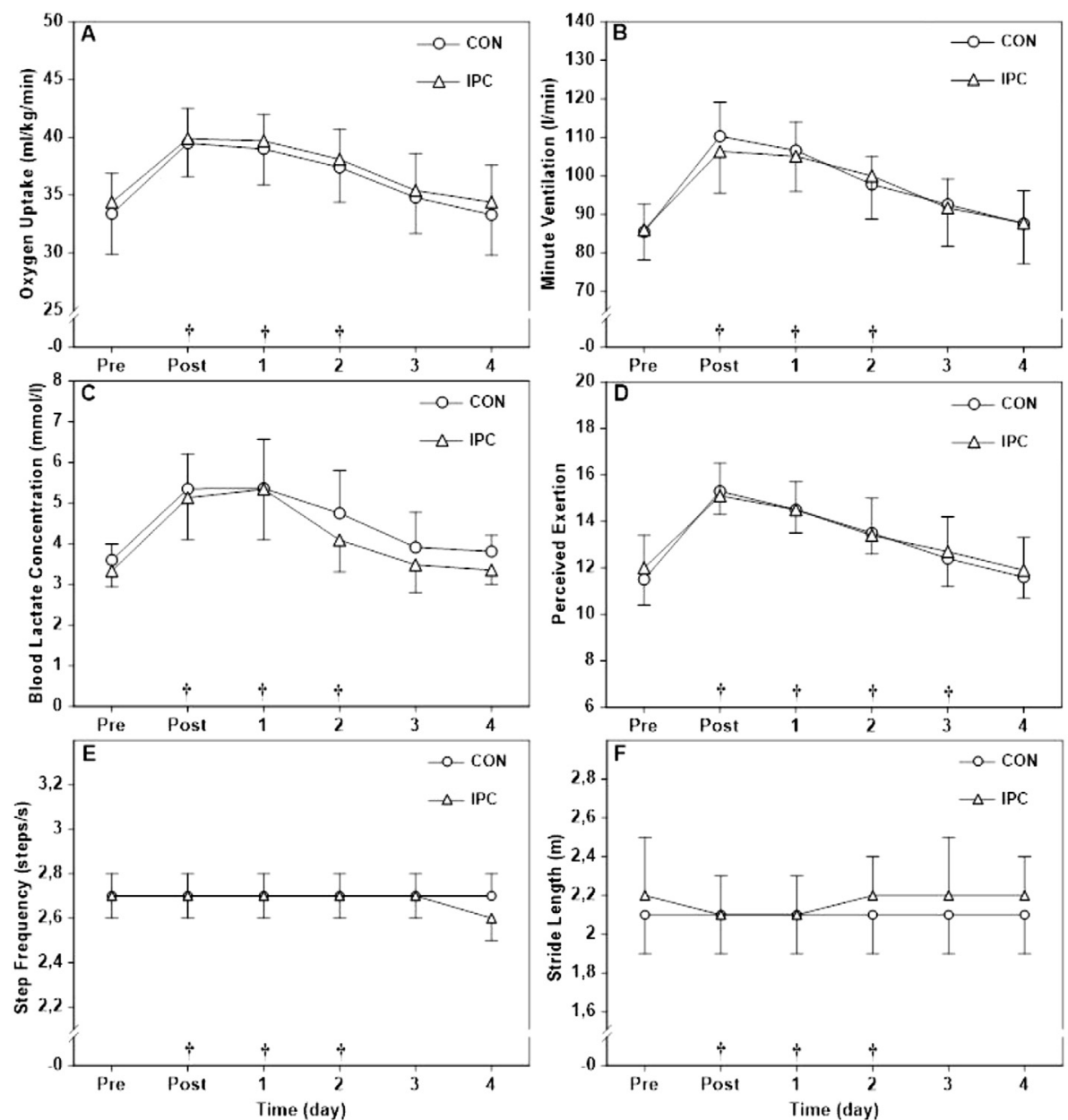

Fig. 3. Changes over time in oxygen uptake (A), minute ventilation (B), blood lactate concentration (C), rate of perceived exertion (D), stride length (E) and step frequency (F). The isometric pre-conditioning group (IPC) performed maximal isometric contractions two days prior to downhill running while the control group (CON) did not perform any pre-conditioning. ${ }^{\dagger} \mathrm{p}<0.05$ compared to baseline values for both groups together.

strength loss following downhill running and; (2) RE parameters were unaffected by IPC. Thus, mechanisms associated with changes in EIMD and RE parameters following a single bout of downhill running seem not be completed shared.

Our hypothesis that IPC would attenuate changes in markers of EIMD following downhill running was partially confirmed by the faster rate of recovery found for muscle soreness and isometric peak torque of the knee extensors in the experimental group. However, IPC did not significantly affect other important markers of EIMD such as serum CK activity or CMJ. CMJ was assessed in the present study to investigate the effects of IPC on muscle function using an exercise condition that is specific to both the pre-conditioning MVIC and downhill running (i.e., closed kinetic chain). However, the obtained data showed that the protective effect conferred by MVIC does not seem to be as dependent on specificity as hypothesized. It is worth mentioning that CMJ performance does not exclusively depend on strength production capacity, but also technique and coordination. This might partially explain our results, as the participants did not have previous experience with this type of activity.

Previous studies showed that IPC significantly attenuated changes in CK, muscle soreness and concentric torque, as well as other markers of EIMD (Chen et al., 2013; Tseng et al., 2016). The vast majority of studies that have investigated IPC did so in the elbow flexors (Chen, Chen, Pearce, \& Nosaka, 2012; Chen, Nosaka, et al., 2012; Chen et al., 2013). Evidence exists showing that upper-limb muscles are more susceptible to EIMD when compared to lower-limb muscles (Chen et al., 2011; Jamurtas et al., 2005) and benefit the most from the repeated bout effect (Lima \& Denadai, 2011). Thus, the difference in responsiveness to EIMD between upper- and lower-limb muscles might explain the dissociation between the findings of the present study and the literature. In fact, we recently proposed a theoretical model hypothesizing that muscles frequently exposed to EIMD (e.g.: knee extensors and plantar flexors) would respond less to IPC due to an already elevated level of protection against EIMD (Lima \& Denadai, 2015). Moreover, we further hypothesized that the volume of pre-conditioning contractions should be considerably greater for muscles that are frequently exposed to EIMD in order to confer any degree of protection.

Indeed, Tseng et al. (2016) recently showed that a high-volume IPC protocol (60 MVICs) blunted changes in force production, CK, muscle soreness and other markers of EIMD following 60 maximal eccentric contractions. However, the IPC protocol proposed by 
Tseng et al. (2016) induced small, yet significant, changes in markers of EIMD (muscle strength, range of motion and plasma CK activity) and was performed two weeks prior to the damaging bout. Although the present study did not assess any marker of EIMD after the IPC protocol, a significant level of protection was conferred by the same volume of contractions used previously on the elbow flexors (Chen, Nosaka, et al., 2012; Chen, Chen, et al., 2012; Chen et al., 2013). As a whole, our data support the hypothesis that the magnitude of IPC protection against EIMD depends on the interaction between muscle group and volume of MVIC (Lima \& Denadai, 2015).

Another factor worth mentioning is the intensity of the damaging bout. Previous studies that investigated IPC used maximal eccentric contractions as damaging activities (Chen, Nosaka, et al., 2012; Chen, Chen, et al., 2012; Chen et al., 2013) while we chose to use downhill running in the present study. Maximal eccentric contractions induce greater strain to the muscular tissue as compared to submaximal eccentric contractions performed during downhill running. This is evidenced by the relatively small increases in serum CK activity identified in the present study (peaking at 250-500 U/1 as compared to $\sim 3000 \mathrm{U} / 1$ in studies that used maximal eccentric contractions), which might partially explain the absence of a significant effect of IPC on serum CK activity. However, in the present study, downhill running induced magnitudes of muscle soreness and strength loss similar to or even greater than those reported in studies that induced damage to the knee extensors with maximal eccentric contractions (Chen et al., 2011; Lima \& Denadai, 2011). Therefore, it seems that, even though downhill running does not inflict great strain to lower limb muscles (which results in de facto tissue damage - indirectly estimated by serum CK activity in the present study), strength production and muscle soreness - which are not exclusively explained by tissue damage, but mostly by compromised excitation-contraction coupling, oxidative stress and inflammation (Hyldahl \& Hubal, 2014) - are similarly affected by this type of damaging bout as compared to maximal eccentric contractions.

Pre-conditioning with MVIC two days prior to downhill running had no significant effects on changes in metabolic and kinematic markers of RE. Both groups presented similar changes in such markers over time, with no effects of group or group vs. time interaction. RE seems to be affected by a wide range of factors such as muscle strength (Støren et al., 2008), muscle-tendon stiffness (Dalleau, Belli, Bourdin, \& Lacour, 1998), anthropometry (Williams \& Cavanagh, 1987), and running kinematics (Moore, 2016). EIMD is also a multifactorial phenomenon that affects many variables that seem to be related to RE. In fact, several studies showed that EIMD leads to impaired RE (Burt, Lamb, Nicholas, \& Twist, 2013; Chen, Nosaka, et al., 2007; Paschalis et al., 2005). However, little is known about the mechanisms that regulate this relationship. Assumpção et al. (2013) reviewed the literature on the relationship between RE and EIMD and concluded that RE is mostly affected by EIMD when assessed at high intensities (80-90\% $\mathrm{VO}_{2} \mathrm{max}$ ), which is why we opted to assess $\mathrm{RE}$ at $80 \% \mathrm{VO}_{2} \mathrm{max}$. Dissociations between the time course of changes in RE markers and changes in force production were also identified by Assumpção et al. (2013). Changes in RE after damaging bouts also appear to be relatively smaller than changes in force production capacity ( $\sim 5$ to $10 \% v s \sim 15$ to $30 \%$, respectively) following damaging bouts, even though it was not the case in the present study.

Two different rationales might explain why IPC did not blunt changes in RE following downhill running in the present study: 1) a low volume of MVIC did not promote a strong enough protective effect against strength loss following downhill running, and RE relies heavily in this factor and/or; 2) downhill running-induced damage affects RE in different ways compared to strength production and other markers of EIMD. The first hypothesis is entirely based on a possible relationship between maximal force production and RE, which has not yet been consistently demonstrated. In fact, a temporal dissociation between recovery of markers of RE and EIMD implies that this relationship is not as strong as previously though (Assumpção et al., 2013). The second hypothesis appears to be the most plausible, therefore. Lima, Nosaka, Pinto, Greco, and Denadai (2017) recently showed that changes in strength and $\mathrm{VO}_{2}$ following downhill running are not significantly correlated. Since both RE and EIMD are multifactorial phenomena, other markers of EIMD that have not yet been studied and compared to changes in RE might help to explain the increase in the cost of running following downhill running.

The number of studies investigating the impact of performing MVIC on prevention and recovery from EIMD is scarse and, to the best of our knowledge, the prophylactic MVIC and the damaging bouts in those studies were performed in similar laboratorial settings (i.e., isokinetic MVIC followed by maximal eccentric contractions performed on the same equipment) (Chen, Nosaka, et al., 2012; Chen, Chen, et al., 2012; Chen et al., 2013; Tseng et al., 2016). However, the aim of the present study was to propose an inexpensive and viable strategy to attenuate EIMD and changes in RE after downhill running.

The choice of IPC as a protective strategy was based on the non-damaging characteristic of this prophylactic strategy. The protective effect conferred by eccentric contractions is well reported in the literature (Hyldahl et al., 2017). However, these strategies induce significant damage to the muscle and require a relatively long period for recovery and adaptation ( $\sim 7$ days). IPC, on the other hand, promotes partial protection against EIMD without damaging the muscle tissue and require as long as two days between the preconditioning bout and the damaging bout to manifest. Some mechanisms that have been proposed to promote the repeated bout effect induced by damaging eccentric contractions are also speculated to promote IPC-induced protection. Augmented expression of genes related to antioxidant response, upregulation of heat-shock proteins, attenuation of neutrophil accumulation and decreased muscular stiffness are mechanisms that seem to be shared by the repeated bout effect and IPC. For mechanistic approaches on IPC and the repeated bout effect, see Lima and Denadai (2015) and Hyldahl et al. (2017), respectively.

A limitation of the present study is the absence of a placebo treatment for the CON group. A possibility exists that the protection conferred by IPC is partially due to neurobiological factors associated with participant's belief, as may be the case for many other recovery strategies (Howatson, Leeder, \& van Someren, 2016). Future research should aim at controlling possible psychobiological biases induced by belief on IPC as a recovery strategy. Furthermore, we consider that the assessment of RE at only one intensity is a limitation of our study. The option of assessing of $\mathrm{RE}$ at $80 \% \mathrm{VO}_{2}$ max was based on a review by Assumpção et al. (2013) which showed that EIMD compromises RE when assessed at high intensities, probably due to the increased contribution of the anaerobic 
metabolism and, consequently, recruitment of a greater pool of fast-twitch fibers (which are predominantly affected by EIMD). However, the manifestation of the $\mathrm{VO}_{2}$ slow component is also likely to occur at this exercise intensity (Xu \& Rhodes, 1999), which could compromise interpretation of $\mathrm{RE}$ in the absence of a $\mathrm{VO}_{2}$ steady state. Therefore we consider the use of a single exercise intensity as a limitation of the present study and that future studies should investigate the effects of IPC on changes in RE at different intensities following DHR. Finally, changes in EIMD markers were not assessed in the period between the MVIC and the downhill run. We cannot state that the IPC protocol did not elicit significant damage because of this limitation. However, the ratings for muscle soreness were zero for all subjects immediately before downhill running and serum CK activity was within normal ranges at the same time point. This indicates that, had any possible damage been induced by the MVIC, it had already subsided two days after it.

\section{Conclusion}

The present study showed that IPC leads to partial protection against changes in two indirect markers of EIMD: muscle soreness and strength loss. Muscle soreness was significantly attenuated and recovered faster when IPC was performed while strength production only recovered faster with no attenuation effect. However, we found that IPC does not seem to affect changes in RE parameters following downhill running. From a clinical point of view, IPC could be performed as an effort to accelerate recovery of muscle function following intense running bouts or attenuate the magnitude of delayed-onset muscle soreness.

\section{Funding}

This work was supported by the São Paulo Research Foundation (FAPESP) (Grant Nos.: 2013/23585-4 and 2016/04909-1).

\section{Appendix A. Supplementary data}

Supplementary data associated with this article can be found, in the online version, at http://dx.doi.org/10.1016/j.humov.2018. 05.002 .

\section{References}

Assumpção, C. O., Lima, L. C. R., Oliveira, F. B., Greco, C. C., \& Denadai, B. S. (2013). Exercise-induced muscle damage and running economy in humans. Scientific World Journal, 2013, 1-11.

Borg, G. (1970). Perceived exertion as an indicator of somatic stress. Scandinavian Journal of Rehabilitation Medicine, 2, 92-98.

Braun, W., \& Paulson, S. (2012). The effects of a downhill running bout on running economy. Research in Sports Medicine, $20,274-285$.

Brentano, M. A., \& Martins Kruel, L. F. (2011). A review on strength exercise-induced muscle damage: Applications, adaptation mechanisms and limitations. Journal of Sports Medicine and Physical Fitness, 51, 1-10.

Burt, D., Lamb, K., Nicholas, C., \& Twist, C. (2013). Effects of repeated bouts of squatting exercise on sub-maximal endurance running performance. European Journal of Applied Physiology, 113, 285-293.

Chen, T. C., Chen, H. L., Lin, M. J., Chen, C. H., Pearce, A. J., \& Nosaka, K. (2013). Effect of two maximal isometric contractions on eccentric exercise-induced muscle damage of the elbow flexors. European Journal of Applied Physiology, 113, 1545-1554.

Chen, T. C., Chen, H. L., Pearce, A. J., \& Nosaka, K. (2012). Attenuation of eccentric exercise-induced muscle damage by preconditioning exercises. Medicine and Science in Sports and Exercise, 44, 2090-2098.

Chen, T. C., Chen, H. L., Wu, C. J., Lin, M. R., Chen, C. H., Wang, L. I., ... Tu, J. H. (2007). Changes in running economy following a repeated bout of downhill running. Journal of Exercise Science \& Fitness, 5, 109-117.

Chen, T. C., Lin, M. J., Chen, H. L., Lai, J. H., Yu, H. I., \& Nosaka, K. (2018). Muscle damage protective effect by two maximal isometric contractions on maximal eccentric exercise of the elbow flexors of the contralateral arm. Scandinavian Journal of Medicine and Science in Sports, 28, 1354-1360.

Chen, T. C., Lin, K. Y., Chen, H. L., Lin, M. J., \& Nosaka, K. (2011). Comparison in eccentric exercise-induced muscle damage among four limb muscles. European Journal of Applied Physiology, 111, 211-223.

Chen, T. C., Nosaka, K., Lin, M. J., Chen, H. L., \& Wu, C. J. (2009). Changes in running economy at different intensities following downhill running. Journal of Sports Sciences, 27, 1137-1144.

Chen, H. L., Nosaka, K., Peake, J., \& Chen, T. C. (2012). Two maximal isometric contractions attenuate the magnitude of eccentric exercise-induced muscle damage. Applied Physiology, Nutrition and Metabolism, 37, 680-689.

Chen, T. C., Nosaka, K., \& Tu, J. H. (2007). Changes in running economy following downhill running. Journal of Sports Sciences, 25 , 55-63.

Clarkson, P. M., \& Hubal, M. J. (2002). Exercise-induced muscle damage in humans. American Journal of Physical Medicine and Rehabilitation, 81, S52-69.

Dalleau, G., Belli, A., Bourdin, M., \& Lacour, J. R. (1998). The spring-mass model and the energy cost of treadmill running. European Journal of Applied Physiology and Occupational Physiology, 77, 257-263.

Denadai, B. S., de Aguiar, R. A., Lima, L. C., Greco, C. C., \& Caputo, F. (2017). Explosive training and heavy weight training are effective for improving running economy in endurance athletes: a systematic review and meta-analysis. Sports Medicine, 47, 545-554. http://dx.doi.org/10.1007/s40279-016-0604-z.

Dias, J. A., Dal Pupo, J., Reis, D. C., Borges, L., Santos, S. G., Moro, A. R., \& Borges, N. G., Jr. (2011). Validity of two methods for estimation of vertical jump height. The Journal of Strength \& Conditioning Research, 25, 2034-2039.

Doma, K., Schumann, M., Leicht, A. S., Heilbronn, B. E., Damas, F., \& Burt, D. (2017). The repeated bout effect of traditional resistance exercises on running performance across 3 bouts. Applied Physiology, Nutrition and Metabolism, 42, 978-985.

Hoogkamer, W., Kipp, S., Spiering, B. A., \& Kram, R. (2016). Altered running economy directly translated to altered distance-running performance. Medicine and Science in Sports and Exercise, 48, 2175-2180.

Howatson, G., Leeder, J., \& van Someren, K. (2016). The BASES expert statement on athletic recovery strategies. The Sport and Exercise Scientist, 48, 6-7.

Hyldahl, R. D., Chen, T. C., \& Nosaka, K. (2017). Mechanisms and mediators of the skeletal muscle repeated bout effect. Exercise and Sport Sciences Reviews, 45, 24-33.

Hyldahl, R. D., \& Hubal, M. J. (2014). Legthening our perspective: Morphological, cellular, and molecular responses to eccentric exercise. Muscle Nerve, 49, 155-170. http://dx.doi.org/10.1002/mus.24077.

Jamurtas, A., Theocharis, V., Tofas, T., Tsiokanos, A., Yfanti, C., Paschalis, V., ... Nosaka, K. (2005). Comparison between leg and arm eccentric exercises of the same relative intensity on indices of muscle damage. European Journal of Applied Physiology, 95, 179-185.

Lacour, J. R., \& Bourdin, M. (2015). Factors affecting the energy cost of level running at submaximal speed. European Journal of Applied Physiology, 115, 651-673.

Lima, L. C. R., Assumpção, C. O., Prestes, J., \& Denadai, B. S. (2015). Consumption of cherries as a strategy to attenuate exercise-induced muscle damage and inflammation in humans. Nutricion Hospitalaria, 32, 1885-1893. 
Lima, L. C. R., \& Denadai, B. S. (2011). The repeated bout effect: a comparison between upper and lower limbs. Motriz, 17, 738-747.

Lima, L. C. R., \& Denadai, B. S. (2015). Attenuation of eccentric exercise-induced muscle damage conferred by maximal isometric contractions: A mini review. Frontiers in Physiology, 6, 300 .

Lima, L.C.R., Nosaka, K., Pinto, R.S., Greco, C.C., \& Denadai, B.S. (2017). What causes a decrease in running economy after downhill running? In Book of Abstracts of the 22nd Annual Congress of the European College of Sport Science, Essen, Germany, 5-8 July 2017.

Moore, I. S. (2016). Is there an economical running technique? A review of modifiable biomechanical factors affecting running economy. Sports Medicine (Auckland, $N$. Z.), 46, 793-807.

Paschalis, V., Koutedakis, Y., Baltzopoulos, V., Mougios, V., Jamurtas, A., \& Theoharis, V. (2005). The effects of muscle damage on running economy in healthy males. International Journal of Sports Medicine, 26, 827-831.

Peñailillo, L., Blazevich, A., Numazawa, H., \& Nosaka, K. (2015). Rate of force development as a measure of muscle damage. Scandinavian Journal of Medicine and Science in Sports, 25, 417-427.

Saunders, P. U., Pyne, D. B., Telford, R. D., \& Hawley, J. A. (2004). Factors affecting running economy in trained distance runners. Sports Medicine (Auckland, N. Z.), 34 , 465-485.

Saunders, P. U., Telford, R. D., Pyne, D. B., Peltola, E. M., Cunningham, R. B., Gorde, C. J., \& Hawley, J. A. (2006). Short-term plyometric training improves running economy in highly trained middle and long distance runners. The Journal of Strength \& Conditioning Research, 20, 947-954.

Støren, O., Helgerud, J., Støa, E. M., \& Hoff, J. (2008). Maximal strength training improves running economy in distance runners. Medicine and Science in Sports and Exercise, 40, 1087-1092.

Tseng, K. W., Tseng, W. C., Lin, M. J., Chen, H. L., Nosaka, K., \& Chen, T. C. (2016). Protective effect by maximal isometric contractions against maximal eccentric exercise-induced muscle damage of the knee extensors. Research in Sports Medicine, 24, 243-256.

Turner, A. M., Owings, M., \& Schwane, J. A. (2003). Improvement in running economy after 6 weeks of plyometric training. The Journal of Strength \& Conditioning Research, 17, 60-67.

Williams, K. R., \& Cavanagh, P. R. (1987). Relationship between distance running mechanics, running economy, and performance. Journal of Applied Physiology, 63, $1236-1245$.

Xu, F., \& Rhodes, E. C. (1999). Oxygen uptake kinetics during exercise. Sports Medicine (Auckland, N. Z.), 27, 313-327. 